\title{
Inhalt des zweiundneunzigsten Bandes.
}

\author{
Erstes Heft (6. April).
}

I. Ueber Cubitus valgus. Von Dr. L. von Lesser, Privatdocenten für Chirurgie in Leipzig. (Hierzu Taf. I.) . . . . . . •

II. Ueber accessorische Nebennieren im Ligamentum latum. Von Prof. I)r. Marchand in Giessen. (Ilierzu Taf. II.) . . . .

III. Uebertragungsversuche von Lepra auf Thiere. Von Dr. Otto Damsch, Privatdocenten und Assistenzarzt an der med. Klinik zu Göttingen. (Hierzu Taf. III.) . . . . . . . . . . .

IV. Beitrag zur Lehre von der doppelten Gebärmutter (Uterus didelphys) nebst besonderer Würdigung der Aetiologie dieser Difformität. Von Dr. P. Kubassow zu St. Petersburg. (Hierzu Taf. IV.) . . . . . . . . . . . . . . . . . . .

V. Noch ein Fall von doppelter Gebärmutter und Scheide (Uterus et Vagina duplices), intra vitam diagnosticirt. Von Demsclben. (Aus der temporären Frauenabtheilung des Krankenhauses St. Nicolai des Wunderthäters in St. Petersburg.) . . . . . .

VI. Beiträge zur Lehre von der örtlichen, zeitlichen und individuellen Disposition des abdominalen Typhus unter Zugrundelegung einer aus den autlichen Listen der Leichenschau des Grossherzogthums Baden für die Jahre 1872-1877 gefertigten Statistik dieser Krankheit. Von Dr. med. Joseph Kugler, pract. Arıt in Königshach in Baden. (Hierzu Taf. V.) (Fortsetzung von Bd.91 S. 563.). . . . . . . . . . . . .

VII. Neue Beiträge zu den Untersuchungen über die Balgdrüsen der Zungenwurzel. (Aus dem pathologischen Institut in Berlin.) Von l)r. Ostmann.

VIII. Ueber die Furchen und Falten der Haut. Von Dr. Lewinski, Docenten an der Universität zu Berlin. (Aus dem pathologischen Institut zu Berlin.) . . . . . . . . . . . . .

IX. Experimentelle Untersuchungen über die Bedeutung des Nervensystems bei Gefässerkrankungen. Von Dr. S. Lew a s chew in St. Petersburg. (Hierzu Taf. VI.) Aus dem klinischen Laboratorium von Prof. S. P. Botkin. . . . . . . . . 1

X. Kleinere Mittheilungen.

1. Eine Beobachtung von angehorener mittlerer vollständiger Halsfistel. Von Stabsarzt Dr. Berg in Ulm. . . .

2. Ueber zwei Fälle von congenitalem Mangel der rechten Niere, complicirt mit Anomalien der Genitalorgane. Von Dr. Paul Guttmann, ärztlichem Director des städtischen Krankenhauses Moabit und Docenten an der Universität Berlin.

3. Carcinosarcoma uteri hei einem Kinde von zwei Jahren. Von Prof. S. Roseustein in Leiden. . . . . . . . 191 


\section{Zweites Heft (2. Mai).}

XI. Beitrag zur Kenntniss der Bleivergiftung. Von Dr. H.v. W y s s in Zürich.

XII. Ueber Spaltbildung am Schädel nach Fractur. Von Gerichtsarzt Prof. Reubold in Würzburg. (Hierzu Taf. VII.). . .

XIII. Zur Aetiologie der Eiterung. (Aus dem pathologischen Institut zu Leipzig.) Von Dr. W. J. Councilman aus Baltimore U. S. A. . . . . . . . . . . . . . . . . .

XIV. Beiträge zur Lehre von der örtlichen, zeitlichen und individuellen Disposition des abdominalen Typhus unter Zugrundelegung einer aus den amtlichen Listen der Leichenschau des Grossherzogthums Baden für die Jahre 1872-1877 gefertigten Statistik dieser Krankheit. Von Dr. med. Joseph Kugler, pract. Arzt in Königsbach in Baden. (Schluss von S. 118.) .

XV. Eine weitverbreitete thierische Mycose. Aus dem pathologischen Institut zu Berlin. Von Max Wolff, Privatdocenten an der Universität. (Hierzu Taf. VIII.) . . . . . . . .

XVI. Ein neuer Fall von Hermaphroditismus (H. spurius masculinus?), am Lebenden beobachtet, mitgetheilt von Prof. Dr. Marchand in Giessen. (Hierzu Taf. IX.) . . . . . . . . . . .

XVII. Ueber die Ostoklastentheorie. Von Dr. Gustav Pommer in Graz. (Hierzu Taf. X.). . . . . . . . . . . 296

XVIII. Kleinere Mittheilungen.

1. Bothriocephalus latus und seine Herkunft. Von Dr. Max Braun in Dorpat. . . . . . . . . . . . . .

2. Nachtrag zu der Abhandlung des Herrn Dr. J. Kugler S. 252. .

\section{Drittes Heft (1. Juni).}

XIX. Ueber die sogenannte „temporäre Form" der acuten atrophischen Spinallähmung Erwachsener. Von Prof. Martin Bernhardt in Berlin. . . . . . . . . . . . . . . . . . .

XX. Ueber Urachus und Urachuscysten. Von J. B. Wutz aus Schwarzhofen (Bayern), e. Assistenten am patholog.-anatom. Institute zu Basel. (Hierzu Taf. XI.). . . . . . . . .

XXI. Untersuchungen über die Circulation in den Nieren. Von Prof. Jul. Cohnheim und Prof. Dr. Charles S. Roy, Director der Brown Institution in London. (Aus dem pathologischen Institut in Leipzig.) (Hierzu Taf. XII-XIII.). . .

XXII. Ueber die Ostoklastentheorie. Von Dr. Gustav Pommer in Graz. (Schluss von S. 360.) . . . . . . . . . . . .

XXIII. Untersuchungen über das chemische und pharmakologische Verhalten der Folia uvae ursi und des Arbutins im Thierkörper. Von Dr. L. Lewin, Docenten der Pharmakologie an der Universităt in Berlin. (Nach Versuchen des Herrn Cand. med. F. Behrend.) . . . . . . . . . . . . . .

XXIV. Beiträge zur pathologischen Anatomie der Syphilis hereditaria der Neugebornen. Von Dr. R. Müller, Bad Nauheim. . .

XXV. Ueber toxische Darmepithelexfoliation. Briefliche Mittheilung an den Herausgeber. Von Prof. R. Böhm in Marburg. 